\title{
Entgegnung zu Bernd Hofmann: Wo lag das Dorf Kyleb des Jahres 1087?, in: Sächsische Heimatblätter 1/2016, S. 60-63
}

Gerhard Billig

Die Aussage: „Bisher konnte das Dorf weder identifiziert noch lokalisiert werden“, ist falsch. Vor rund fünfzig Jahren wurde im Kulturspiegel „Meißner Heimat" von Kurt Biehayn die Lage des Ortes mit Klipphausen interpretiert, und dazu Widerspruch geäußert, der einschloss, dass ein Rückschluss auf eine so frühe einmalige Erwähnung insgesamt fragwürdig sei. Das ,Klipp' vor ,hausen' hat mit Kyleb nichts zu tun. Der gesamte Name wurde nach dem Schlossneubau 1554 von adliger Seite auf das Rittergut übertragen. Zuvor hieß der Ort Rüdigsdorf/ Röhrsdorf, eine typische Namengebung des Landesausbaus, in der Bedeutung „Dorf eines Rüdiger“. Das 11. Jahrhundert wird damit nicht berührt.

Cosmas berichtet in der Böhmischen Chronik von der Niederschlagung einer Revolte des Dorfes im Zusammenhang der Verlegung der Burg Guozdec. Man kann rückschließend die Anlage am Burgberg Niederwartha lokalisieren. Direkt und zweifelsfrei gelingt das aber ebenfalls nicht. Cosmas, der von Kyleb berichtet, spricht immer von Guozdec. Die Quellen der Mark Meißen und der Einrichtungen des Erzbistums Magdeburg und des Bistums Merseburg verwenden vorzügig Wos und Wosice. Dass es sich mit hoher Wahrscheinlichkeit um Niederwartha handelt, geht aus der letzten Erwähnung vom Burgward Wozice 1140 hervor. Die Urkunde nennt Dörfer des frühen Landesausbaus im Süden von Niederwartha, das so im Bild der topographischen Lage als Ausgangspunkt der Siedelbewegung erscheint. Die Burgwarderwähnungen von 1046 bis 1140 bezeugen die Namen Woz, Wosice, Guozdec.

Wartha erscheint erstmals 1205. Die Ortslagen von Nieder- und Oberwartha berühren außerdem die Wüstung Grunau (1485). Damit zeigt sich ein Namenwandel und wohl auch Doppelung im Verlauf des 11. und 12. Jahrhunderts, erkennbar aus der Reihung und Wiederholung der Namenserwähnungen. Im Vergleich zeigt sich die geringe Chance der Lokalisierung der einmaligen Erwähnung Kyleb.

Die Chronik des Cosmas kennt die politische Organisation der von den Ottonen angelegten Marken nördlich des Erzgebirges und Lausitzer Gebirges nicht oder unzureichend. Entsprechende Namen fehlen. Selbst der Begriff „Mark Meißen“ bleibt unerwähnt. Cosmas nennt das Gebiet nördlich von Böhmen „Srbia“, das bedeutet so viel wie „Sorbenland“. Auch den Burgward als kleinste Verwaltungseinheit der Mark kennt Cosmas nicht. Er spricht von Guozdec als castrum oder castellum, das heißt schlicht Burg/Befestigung. Für einen Burgbezirk steht allgemein der Begriff civitas, den man als Burgward vergleichend auffassen könnte. Auch er fehlt bei Cosmas. So zeigt der Doppelname für Burg und Vorort Guozdec/Woz möglicherweise ähnliches für das nur einmalig genannte Kyleb. Damit ist der Versuch der Lokalisierung durch diese Eigenart der Überlieferung stark belastet und eingeschränkt.
Die überregionale Bedeutung der Burg Guozdec, die durch ihre Verlegung unterstrichen wird, liegt in dem Kampf Kaiser Heinrichs IV. gegen die aufständische Fürstenopposition, in der der Markgraf Ekbert II. von Meißen eine herausragende Rolle spielte. Heinrich IV. übertrug dem Böhmenherzog Vratislav den Gau Nisan mit der Aufgabe, Ekbert in Meißen auszuschalten. Auch der kaisertreue Schwiegersohn von Vratislav, Wiprecht von Groitzsch, besaß Hoheitsrechte in Nisan. Guozdec-Niederwartha war Ausgangsposition im Kampf gegen Meißen. Mit dem Tod Ekberts 1090 erlosch diese strategrsche Bedeutung. Das fordert einen Vergleich mit dem archäologischen Befund der Wehranlagen von Niederwartha.

Die Flur Niederwartha umfasst drei in der Zeitstellung unterschiedliche mittelalterliche Wehranlagen: Den Burgberg, den Böhmerwall und den Heiligen Hain. Eine umfassende wissenschafliche Ausgrabung und damit verbindliche Ergebnisse stehen aus. Zufall und bauliche Eingriffe erbrachten Lesefunde, die in Verbindung mir der Gestalt der Wehranlagen relativ sichere Rückschlüsse erlauben. Auf dem Burgberg befand sich im Zweiten Weltkrieg eine Flakstellung. Die Mannschaftsbaracke wurde nach dem Krieg als Wochenendhaus genutzt und ausgebaut, in der eigentlichen Stellung entstand eine Sauna mit Pool. Auf dem Turmhügel des Heligen Hain wurde ein Wochenendhaus errichtet. Es bestehen also erhebliche Störungen.

Die Verlegung der Burg Guozdec kann man mit hoher Wahrscheinlichket zwischen Burgberg und Böhmerwall erkennen. Das zeigt nicht allein der Flurname „Böhmerwall“ an. Er ist sicherlich im Anwohnerbereich in Kenntnis der historischen Überlieferung entstanden. Übereinstimmend sprechen die Gestalt der Wehrbauten und die Scherbenfunde für jüngere Zeitstellung gegenüber dem Burgberg. Die archäologischen Funde zeigen jedoch eine wesentliche Ergänzung der schriftlichen Nachricht. Der Böhmerwall wurde offenbar im ausgehenden 11. Jahrhundert errichtet, aber der Burgberg blieb, wie die Scherben anzeigen, weiter bestehen. Die „Verlegung“ bestand in einem Neubau in der Nähe an einer Stelle von höherer Sicherheit. Die alte Burg wurde aber nicht aufgegeben, sondern blieb als Nebenwerk bestehen. Das bedeutete keine bloße Verlegung, sondern fortifikatorische Verstärkung.

Der Turmhügel des Heiligen Hains (der Flurname entstand wohl unter Einfluss der Romantik) kann mit dem Herrensitz von 1205 in Verbindung gesehen werden. Er liegt am Hals eines geräumigen Talsporns. Dieser ist insgesamt besiedelt gewesen. Eindeutige Scherbenfunde weisen die Belegung vor Errichtung des Turmhügels seit Anfang des 12. Jahrhunderts aus. Ob diese Siedlung als Vorgänger des Turmhügels befestigt war, bleibt ungewiss. Es zeigt sich, Siedlungsmuster und Befestigungen in 
und um Niederwartha unterlagen bedeutsamen einschneidendem Wandel.

Wenn Hofmann zitiert: „Allda wollte König Wratislaus ein wüstes Schloß (Guozdec) unweit der Stadt Meißen gelegen, wieder aufbauen, aber die Bauern aus einem großen Dorfe mit Namen Kyleb wollten dies verhindern. König Wratislaus aber wollte sich dafür rächen und sandte seinen Sohn Bretislav dahin, dass er es retten und den Maurern, Steinhauern, Zimmerleuten und Dachdeckern kein Hindernis bei ihrer Arbeit in den Weg gelegt werden sollte...", so handelt es sich um abwegige Vorstellungen des ausgehenden 16. Jahrhunderts und keinesfalls um historisch beweisfähige Quellenaussage. Richtungsweisende Ausgangsposition bildet immer nur die Originalquelle, d. h. die Chronik des Cosmas in Mittellatein, zu der leider eine autorisierte Übersetzung fehlt (Druck in Monumenta Germaniae historica. Scriptores rerum Germanicarum: Die Chronik der Böhmen des Cosmas von Prag Pragensis [Cosmae Pragensis Chronica Boemorum], hrsg. v. B. Bretholz, Berlin 1923, Nachdruck München 1980). Aus dem Zusammenhang der Quelle geht hervor, dass es sich bei Revolte und Vergeltung in Kyleb anlässlich der Verlegung von Guozdec um eine örtliche Bewegung handelte, die nicht den gesamten Gau Nisan betraf. Deshalb suchte Biehayn den Ort in der Nähe von Niederwartha; deshalb entspricht der Rahmen der Erörterungen von Bernd Hofmann nicht den historischen Zusammenhängen des ausgehenden 11. Jahrhunderts. Es geht um lokale Vorgänge im Nordwesten des Gaues Nisan. Dort liegen die Burgwarde Woz/Guozdec/Niederwartha, Bries-nitz und Weißeritz, die enger miteinander verbunden sind, weil sie mit hoher Wahrscheinlichkeit eine gemeinsame Kirche in Briesnitz besaßen. Kleba scheidet schon damit aus, denn es gehörte zu Dohna oder zu einem fraglichen hypothetischen Burgward Lockwitz.
Die Linguistik Hofmanns ist keineswegs wissenschaftlich seriös. Das Ventilieren der Konsonantenfolge erscheint als individuelle Spielerei. Die Sprachwissenschaft geht von der Ganzheit des Namens aus, und dazu gehören die Vokale. Auch anderweitig negiert und übergeht Hofmann die Ergebnisse von Ernst Eichler und Hans Walther zu den Ortsnamen von Nisan und Daleminze und damit auch alle Erkenntnisse und Methoden der $\mathrm{Na}-$ menforschung an der Universität Leipzig, sowie die Veröffentlichungsreihe Deutsch-slawische Forschungen. Das Historische Ortsnamenbuch von Sachsen zitiert Hofmann, aber die Feststellung zu Kleba übergeht er. Dort werden zwei Deutungsmögtichkeiten vorgestellt, eine deutsche und eine slawische, mit dem Schluss: „Eine Entscheidung läßt sich nicht treffen." Damit ist jede sichere Beziehung zwischen Kleba und Kyleb ausgeschlossen. Zwischen dem ausgehenden 11. Jahrhundert und der Ersterwähnung von Kleba 1288 liegen früher und großer Landesausbau mit grundlegender Veränderung der Siedlungslandschaft. Neuerungen des Landesausbaus wirkten nachhaltig auf das Altsiedelgebiet mit Verlegung und Erweiterungen von Orten zurück. Danach folgten Wüstungsvorgänge. Rückschlüsse vom gegenwärtigen, seit dem 19. Jahrhundert dokumentierten Siedlungsbild sind eingeschränkt und grundsätzlich zu hinterfragen. So bleibt zu Kyleb folgendes Resumee: Es handelt sich um eine nicht lokalisierbare frühe Wüstung im Nordwesten des Altsiedelgebietes der Dresdner Elbtalweitung, auf dem linken Ufer der Elbe zwischen Tännichtgrund und dem in historischer Zeit stark pendelnden Elbebogen, der heute durch die Flügelwegbrücke markiert wird.

Auf Literaturhinweis wird verzichtet, weil die Angaben bei Hofmann zum eigentlichen Problem Lücken aufweisen. Die zu füllen, kann hier nicht als notwendige Aufgabe verbindlich erscheinen.

\section{Autor}

Prof. Dr. Gerhard Billig Dresden

\section{IMPRESSUM Sächsische Heimatblätter}

Unabhängige Zeitschrift für Sächsische Geschichte, Landeskunde, Natur und Umwelt

Herausgeber: Dr. Lars-Arne Dannenberg und Dr. Matthias Donath in Zusammenarbeit mit einem Redaktionsbeirat

Anschrift: Zentrum für Kultur//Geschichte, Dorfstraße 3, 01665 Niederjahna

shb@zkg-dd.de

Redaktion: Dr. Lars-Arne Dannenberg, Dr. Matthias Donath, Dr. Romy Petrick

Redaktionsbeirat: $\quad$ Dr. Jens Beutmann, Prof. Dr. Enno Bünz, Prof. Dr. Thomas Bürger, Günter Donath, Dr. Heinrich Douffet, Prof. Dr. Angelica Dülberg, Anneliese Eschke, Dr.-Ing. Gerhard Glaser, Klaus Gumnior, Dr. Konstantin Hermann, Dr. Wolfgang Hocquél, Prof. Dr. Uwe Ulrich Jäschke, Dr. Igor Jenzen, Prof. Dr. Winfried Müller, Wolfgang Schwabenicky, Dr. André Thieme, Dr. Ralf Thomas, Dr. Michael Wetzel, Dr. Peter Wiegand

Herstellung: $\quad$ Redaktions- und Verlagsgesellschaft Elbland mbH Meißen

Erscheinungsweise: Vierteljährlich

Bezugsbedingungen:

Die Zeitschrift ist im Jahresabonnement (4 Ausgaben) zum Preis von 30,00 € inklusive Mwst., Versand und Porto zu beziehen. Die Aufnahme eines Abonnements ist jederzeit möglich bei anteiligem Abopreis. Kündigungen müssen schriftlich bis zum 15. November eines Jahres für das Folgejahr an das Zentrum für Kultur//Geschichte, Dorfstraße 3, 01665 Niederjahna, eingegangen sein. Im freien Verkauf kostet das Einzelheft zwischen 8,50€ und 12,00€.

Für den Inhalt der Beiträge sowie die Abbildungsrechte zeichnen jeweils die Autoren verantwortlich. Jede Verwertung der Inhalte außerhalb der Grenzen des Urheberrechts ist unzulässig. Nachdruck, auch auszugsweise, darf nur mit Zustimmung der Herausgeber erfolgen.

Titelfoto: Dom zu Meißen, Georgskapelle, Tür mit den Wappen Sachsens und Polens. Foto: Matthias Donath Der Druck dieser Publikation wurde durch das Sächsische Staatsministerium des Innern gefördert. 